\title{
Existence and Global Behavior of Positive Solutions for Some Fourth-Order Boundary Value Problems
}

\author{
Ramzi S. Alsaedi \\ Department of Mathematics, College of Sciences and Arts, King Abdulaziz University, Rabigh Campus, P.O. Box 344, \\ Rabigh 21911, Saudi Arabia
}

Correspondence should be addressed to Ramzi S. Alsaedi; ramzialsaedi@yahoo.co.uk

Received 10 November 2013; Accepted 27 February 2014; Published 31 March 2014

Academic Editor: Samir Saker

Copyright (C) 2014 Ramzi S. Alsaedi. This is an open access article distributed under the Creative Commons Attribution License, which permits unrestricted use, distribution, and reproduction in any medium, provided the original work is properly cited.

We establish the existence and uniqueness of a positive solution to the following fourth-order value problem: $u^{(4)}(x)=a(x) u^{\sigma}(x)$, $x \in(0,1)$ with the boundary conditions $u(0)=u(1)=u^{\prime}(0)=u^{\prime}(1)=0$, where $\sigma \in(-1,1)$ and $a$ is a nonnegative continuous function on $(0,1)$ that may be singular at $x=0$ or $x=1$. We also give the global behavior of such a solution.

\section{Introduction}

The purpose of this paper is to study the existence and uniqueness with a precise global behavior of a positive solution $u \in C^{4}((0,1)) \cap C([0,1])$ for the following fourthorder two-point boundary value problem:

$$
\begin{gathered}
u^{(4)}(x)=a(x) u^{\sigma}(x), \quad x \in(0,1), \\
u(0)=u(1)=u^{\prime}(0)=u^{\prime}(1)=0,
\end{gathered}
$$

where $-1<\sigma<1$ and $a$ is a nonnegative continuous function on $(0,1)$ that may be singular at $x=0$ or $x=1$ and satisfies some hypotheses related to the class of Karamata regularly varying functions.

There have been extensive studies on fourth-order boundary value problems with diverse boundary conditions via many methods; see, for example, [1-9] and the references therein.

A naturel motivation for studying higher order boundary value problems lies in their applications. For example, it is well known that the deformation of an elastic beam in equilibrium state, whose both ends clamped, can be described by fourth-order boundary value problem

$$
\begin{gathered}
u^{(4)}(x)=g(x, u(x)), \quad x \in(0,1), \\
u(0)=u(1)=u^{\prime}(0)=u^{\prime}(1)=0 .
\end{gathered}
$$

Our aim in this paper is to give a contribution to the study of these problems by exploiting the properties of the Karamata class of functions.

To state our result, we need some notations. We denote by $C([0,1])$ the set of all continuous functions $f$ on $[0,1]$, and we will use $\mathscr{K}$ to denote the set of Karamata functions $L$ defined on $(0, \eta]$ by

$$
L(t):=c \exp \left(\int_{t}^{\eta} \frac{z(s)}{s} d s\right)
$$

for some $\eta>1$, where $c>0$ and $z \in C([0, \eta])$ such that $z(0)=0$. It is clear that a function $L$ is in $\mathscr{K}$ if and only if $L$ is a positive function in $C^{1}((0, \eta])$ such that

$$
\lim _{t \rightarrow 0^{+}} \frac{t L^{\prime}(t)}{L(t)}=0 .
$$

For two nonnegative functions $f$ and $g$ defined on a set $S$, the notation $f(x) \approx g(x), x \in S$, means that there exists $c>0$ such that $(1 / c) f(x) \leq g(x) \leq c f(x)$, for all $x \in S$. We denote by $x^{+}=\max (x, 0), x \wedge t=\min (x, t), x \vee t=\max (x, t)$, for $x, t \in \mathbb{R}$, and $B^{+}((0,1))$ the set of all measurable functions on $(0,1)$.

Throughout this paper, we assume that $a$ is nonnegative on $(0,1)$ and satisfies the following condition:

$$
\begin{aligned}
& \left(H_{0}\right) a \in C((0,1)) \text { such that for } t \in(0,1) \\
& \qquad a(t) \approx t^{-\lambda} L_{1}(t)(1-t)^{-\mu} L_{2}(1-t),
\end{aligned}
$$


where $\lambda \leq 3+\sigma, \mu \leq 3+\sigma, L_{1}, L_{2} \in \mathscr{K}$ satisfying

$$
\int_{0}^{\eta} t^{2+\sigma-\lambda} L_{1}(t) d t<\infty, \quad \int_{0}^{\eta} t^{2+\sigma-\mu} L_{2}(t) d t<\infty .
$$

In the sequel, we introduce the function $\theta_{\lambda, \mu}$ defined on $(0,1)$ by

$$
\begin{aligned}
\theta_{\lambda, \mu}(x)= & x^{\min (2,(4-\lambda) /(1-\sigma))}\left(\widetilde{L}_{1}(x)\right)^{1 /(1-\sigma)} \\
& \times(1-x)^{\min (2,(4-\lambda) /(1-\sigma))}\left(\widetilde{L}_{2}(1-x)\right)^{1 /(1-\sigma)},
\end{aligned}
$$

where

$$
\begin{aligned}
& \widetilde{L}_{1}(x)= \begin{cases}1 & \text { if } \lambda<2(1+\sigma), \\
\int_{x}^{\eta} \frac{L_{1}(s)}{s} d s & \text { if } \lambda=2(1+\sigma), \\
L_{1}(x) & \text { if } 2(1+\sigma)<\lambda<3+\sigma, \\
\int_{0}^{x} \frac{L_{1}(s)}{s} d s & \text { if } \lambda=3+\sigma,\end{cases} \\
& \widetilde{L}_{2}(x)= \begin{cases}1 & \text { if } \mu<2(1+\sigma), \\
\int_{x}^{\eta} \frac{L_{2}(s)}{s} d s & \text { if } \mu=2(1+\sigma), \\
L_{2}(x) & \text { if } 2(1+\sigma)<\mu<3+\sigma, \\
\int_{0}^{x} \frac{L_{2}(s)}{s} d s & \text { if } \mu=3+\sigma .\end{cases}
\end{aligned}
$$

Our main result is the following.

Theorem 1. Let $\sigma \in(-1,1)$ and assume that a satisfies $\left(H_{0}\right)$. Then, problem (1) has a unique positive solution $u \in$ $C^{4}((0,1)) \cap C([0,1])$ satisfying for $x \in(0,1)$

$$
u(x) \approx \theta_{\lambda, \mu}(x)
$$

This paper is organized as follows. Some preliminary lemmas are stated and proved in the next section, involving some already known results on Karamata functions. In Section 3, we give the proof of Theorem 1 .

\section{Technical Lemmas}

To let the paper be self-contained, we begin this section by recapitulating some properties of Karamata regular variation theory. The following is due to $[10,11]$.

Lemma 2. The following assertions hold.

(i) Let $L \in \mathscr{K}$ and $\varepsilon>0$; then, one has

$$
\lim _{t \rightarrow 0^{+}} t^{\varepsilon} L(t)=0
$$

(ii) Let $L_{1}, L_{2} \in \mathscr{K}$ and let $p \in \mathbb{R}$. Then, one has $L_{1}+L_{2} \in$ $\mathscr{K}, L_{1} L_{2} \in \mathscr{K}$, and $L_{1}^{p} \in \mathscr{K}$.
Example 3. Let $m$ be a positive integer. Let $c>0$, let $\left(\mu_{1}, \mu_{2}, \ldots, \mu_{m}\right) \in \mathbb{R}^{m}$, and let $d$ be a sufficiently large positive real number such that the function

$$
L(t)=c \prod_{k=1}^{m}\left(\log _{k}\left(\frac{d}{t}\right)\right)^{\mu_{k}}
$$

is defined and positive on $(0, \eta]$, for some $\eta>1$, where $\log _{k} x=\log \circ \log \cdots \cdots \circ \log x$ ( $k$ times). Then, $L \in \mathscr{K}$.

Applying Karamata's theorem (see $[10,11]$ ), we get the following.

Lemma 4. Let $\mu \in \mathbb{R}$ and let $L$ be a function in $\mathscr{K}$ defined on $(0, \eta]$. One has the following:

(i) if $\mu<-1$, then $\int_{0}^{\eta} s^{\mu} L(s) d s$ diverges and $\int_{t}^{\eta} s^{\mu} L(s) d s \sim_{t \rightarrow 0^{+}}-\left(t^{1+\mu} L(t) /(\mu+1)\right)$;

(ii) if $\mu>-1$, then $\int_{0}^{\eta} s^{\mu} L(s) d s$ converges and $\int_{0}^{t} s^{\mu} L(s) d s \sim_{t \rightarrow 0^{+}}\left(t^{1+\mu} L(t) /(\mu+1)\right)$.

Lemma 5 (see [12] or [13]). Let $L \in \mathscr{K}$ be defined on $(0, \eta]$. Then, one has

$$
\lim _{t \rightarrow 0^{+}} \frac{L(t)}{\int_{t}^{\eta}(L(s) / s) d s}=0 .
$$

If further $\int_{0}^{\eta}(L(s) / s) d s$ converges, then one has

$$
\lim _{t \rightarrow 0^{+}} \frac{L(t)}{\int_{0}^{t}(L(s) / s) d s}=0 .
$$

Remark 6. Let $L \in \mathscr{K}$ be defined on $(0, \eta]$; then, using (4) and (12), we deduce that

$$
t \rightarrow \int_{t}^{\eta} \frac{L(s)}{s} d s \in \mathscr{K} .
$$

If further $\int_{0}^{\eta}(L(s) / s) d s$ converges, we have by (12) that

$$
t \longrightarrow \int_{0}^{t} \frac{L(s)}{s} d s \in \mathscr{K} .
$$

Lemma 7. Given that $f \in C([0,1])$, then the unique continuous solution of

$$
\begin{gathered}
u^{(4)}(x)=f(x), \quad x \in(0,1), \\
u(0)=u(1)=u^{\prime}(0)=u^{\prime}(1)=0
\end{gathered}
$$

is given by

$$
u(x)=G f(x):=\int_{0}^{1} G(x, t) f(t) d t
$$

where

$G(x, t)$

$$
=\frac{1}{6}(x \wedge t)^{2}(1-x \vee t)^{2}[3(x \vee t)-(x \wedge t)(1+2(x \vee t))]
$$

is Green's function for the boundary value problem (16). 
Remark 8. For $x, t \in(0,1)$, we have $G(1-x, 1-t)=G(x, t)$.

In the following, we give some estimates on the Green function $G(x, t)$ that will be used later.

Proposition 9. On $(0,1) \times(0,1)$, one has the following:

(i) $(1 / 3)(x \wedge t)^{2}(1-x \vee t)^{2}(x \vee t)(1-x \wedge t) \leq G(x, t) \leq$ $(1 / 2)(x \wedge t)^{2}(1-x \vee t)^{2}(x \vee t)(1-x \wedge t)$

(ii) $(1 / 3) x^{2}(1-x)^{2} t^{2}(1-t)^{2} \leq G(x, t) \leq(1 / 2) x(1-x) t^{2}(1-$ $t)^{2}$.

Proof. (i) It follows from the fact that for $x, t \in(0,1) \times(0,1)$ we have

$$
\begin{aligned}
2(x \vee t)(1-x \wedge t) & \leq 3(x \vee t)-(x \wedge t)(1+2 x \vee t) \\
& \leq 3(x \vee t)(1-x \wedge t)
\end{aligned}
$$

(ii) Since for $x, t \in(0,1)$ we have $x^{2}(1-x)^{2} t^{2}(1-t)^{2} \leq$ $(x \wedge t)^{2}(1-x \vee t)^{2}(x \vee t)(1-x \wedge t)$, the result follows from (i).

As a consequence of the assertion (ii) of Proposition 9, we obtain the following.

Corollary 10. Let $f \in B^{+}((0,1))$ and put $G f(x):=$ $\int_{0}^{1} G(x, t) f(t) d t$, for $x \in(0,1]$.

Then,

$$
\begin{gathered}
\text { Gf }(x)<\infty \\
\text { for } x \in(0,1) \quad \text { iff } \int_{0}^{1} t^{2}(1-t)^{2} f(t) d t<\infty .
\end{gathered}
$$

Proposition 11. Let $f$ be a measurable function such that the function $t \rightarrow t^{2}(1-t)^{2} f(t)$ is continuous and integrable on $(0,1)$. Then, $G f$ is the unique solution in $C^{4}((0,1)) \cap C([0,1])$ of the problem

$$
\begin{gathered}
u^{(4)}(x)=f(x), \quad x \in(0,1), \\
u(0)=u(1)=u^{\prime}(0)=u^{\prime}(1)=0 .
\end{gathered}
$$

Proof. From Corollary 10, the function $G f$ is defined on $(0,1)$ and, by Proposition 9, we have

$$
G(f)(x) \leq \frac{1}{2} x(1-x) \int_{0}^{1} t^{2}(1-t)^{2}|f(t)| d t
$$

Now, since $t \rightarrow t^{2} f(t)$ is integrable near 0 and $t \rightarrow(1-$ $t)^{2} f(t)$ is integrable near 1 , then, for $x \in(0,1)$, we have

$$
\begin{aligned}
G f(x)= & \frac{1}{2} x(1-x)^{2} \int_{0}^{x} t^{2} f(t) d t \\
& +\frac{1}{2} x^{2} \int_{x}^{1} t(1-t)^{2} f(t) d t
\end{aligned}
$$

$$
\begin{aligned}
& -\frac{1}{6}(1+2 x)(1-x)^{2} \int_{0}^{x} t^{3} f(t) d t \\
& -\frac{1}{6} x^{3} \int_{x}^{1}(1+2 t)(1-t)^{2} f(t) d t .
\end{aligned}
$$

This gives

$$
\begin{aligned}
(G f)^{\prime}(x)= & \frac{1}{2}(1-3 x)(1-x) \int_{0}^{x} t^{2} f(t) d t \\
& +x \int_{x}^{1} t(1-t)^{2} f(t) d t+x(1-x) \int_{0}^{x} t^{3} f(t) d t \\
& -\frac{1}{2} x^{2} \int_{x}^{1}(1+2 t)(1-t)^{2} f(t) d t
\end{aligned}
$$

$$
\begin{aligned}
(G f)^{\prime \prime}(x)= & (3 x-2) \int_{0}^{x} t^{2} f(t) d t \\
& +\int_{x}^{1} t(1-t)^{2} f(t) d t+(1-2 x) \int_{0}^{x} t^{3} f(t) d t \\
& -x \int_{x}^{1}(1+2 t)(1-t)^{2} f(t) d t
\end{aligned}
$$$$
(G f)^{\prime \prime \prime}(x)=\int_{0}^{x}\left(3 t^{2}-2 t^{3}\right) f(t) d t
$$$$
-\int_{x}^{1}(1+2 t)(1-t)^{2} f(t) d t
$$

$(G f)^{(4)}(x)=f(x)$.

Moreover, we have $G f(0)=G f(1)=(G f)^{\prime}(0)=$ $(G f)^{\prime}(1)=0$.

Finally, we prove the uniqueness. Let $u, v \in C^{4}((0,1)) \cap$ $C([0,1])$ be two solutions of $(21)$ and put $w=v-u$. Then, $w \in C^{4}((0,1)) \cap C([0,1])$ and $w^{(4)}=0$. Hence, it follows that $w(x)=a x^{3}+b x^{2}+c x+d$. Using the fact that $w(0)=w(1)=$ $w^{\prime}(0)=w^{\prime}(1)=0$, we conclude that $w=0$ and so $u=v$.

In the sequel, we assume that $\beta \leq 3$ and $\gamma \leq 3$ and we put

$$
b(t)=t^{-\beta} L_{3}(t)(1-t)^{-\gamma} L_{4}(1-t),
$$

where $L_{3}, L_{4} \in \mathscr{K}$ satisfy

$$
\int_{0}^{\eta} t^{2-\beta} L_{3}(t) d t<\infty, \quad \int_{0}^{\eta} t^{2-\gamma} L_{4}(t) d t<\infty
$$

So, we aim to give some estimates on the potential function $G b(x)$. 
We define the Karamata functions $\psi_{\beta}, \phi_{\gamma}$ by

$$
\begin{aligned}
& \psi_{\beta}(x)= \begin{cases}\int_{0}^{x} \frac{L_{3}(t)}{t} d t & \text { if } \beta=3, \\
L_{3}(x) & \text { if } 2<\beta \leq 3, \\
\int_{x}^{\eta} \frac{L_{3}(t)}{t} d t & \text { if } \beta=2, \\
1 & \text { if } \beta<2,\end{cases} \\
& \phi_{\gamma}(x)= \begin{cases}\int_{0}^{x} \frac{L_{4}(t)}{t} d t & \text { if } \gamma=3, \\
L_{4}(x) & \text { if } 2<\gamma<3, \\
\int_{x}^{\eta} \frac{L_{4}(t)}{t} d t & \text { if } \gamma=2, \\
1 & \text { if } \gamma<2 .\end{cases}
\end{aligned}
$$

Then, we have the following.

Proposition 12. For $x \in(0,1)$,

$$
G b(x) \approx x^{\min (2,4-\beta)}(1-x)^{\min (2,4-\gamma)} \psi_{\beta}(x) \phi_{\gamma}(1-x) .
$$

Proof. Using Proposition 9, we have

$$
\begin{aligned}
G b(x) \approx & \int_{0}^{1}(1-t)^{-\gamma} t^{-\beta}(x \wedge t)^{2} \\
& \quad \times(1-x \vee t)^{2}(x \vee t)(1-x \wedge t) L_{3}(t) L_{4}(1-t) d t \\
\approx & x(1-x)^{2} \int_{0}^{x}(1-t)^{1-\gamma} t^{2-\beta} L_{3}(t) L_{4}(1-t) d t \\
& +x^{2}(1-x) \int_{x}^{1}(1-t)^{2-\gamma} t^{1-\beta} L_{3}(t) L_{4}(1-t) d t \\
= & x(1-x)^{2} I(x)+x^{2}(1-x) J(x) .
\end{aligned}
$$

For $0<x \leq 1 / 2$, we have $I(x) \approx \int_{0}^{x} t^{2-\beta} L_{3}(t) d t$. So, using Lemma 4 and hypothesis (26), we deduce that

$$
I(x) \approx \begin{cases}\int_{0}^{x} \frac{L_{3}(t)}{t} d t & \text { if } \beta=3, \\ x^{3-\beta} L_{3}(x) & \text { if } \beta<3 .\end{cases}
$$

Now, we have

$$
\begin{aligned}
J(x) & \approx \int_{x}^{1 / 2} t^{1-\beta} L_{3}(t) d t+\int_{1 / 2}^{1}(1-t)^{2-\gamma} L_{4}(1-t) d t \\
& \approx 1+\int_{x}^{1 / 2} t^{1-\beta} L_{3}(t) d t .
\end{aligned}
$$

This implies by Lemma 4 that

$$
J(x) \approx \begin{cases}x^{2-\beta} L_{3}(x) & \text { if } 2<\beta \leq 3, \\ \int_{x}^{\eta} \frac{L_{3}(t)}{t} d t & \text { if } \beta=2, \\ 1 & \text { if } \beta<2 .\end{cases}
$$

Hence, it follows by Lemma 5 and hypothesis (26) that, for $0<x \leq 1 / 2$, we get

$$
G b(x) \approx \begin{cases}x \int_{0}^{x} \frac{L_{3}(t)}{t} d t & \text { if } \beta=3, \\ x^{4-\beta} L_{3}(x) & \text { if } 2<\beta<3, \\ x^{2} \int_{x}^{\eta} \frac{L_{3}(t)}{t} d t & \text { if } \beta=2 \\ x^{2} & \text { if } \beta<2,\end{cases}
$$

That is, for $0<x \leq 1 / 2$,

$$
G b(x) \approx x^{\min (2,4-\beta)} \psi_{\beta}(x) .
$$

Now, since $G(1-x, 1-t)=G(x, t)$, we use similar arguments as above applied to $L_{4}$ instead of $L_{3}$ to obtain

$$
G b(x) \approx(1-x)^{\min (2,4-\gamma)} \phi_{\gamma}(1-x) \quad \text { for } \frac{1}{2} \leq x \leq 1 .
$$

This together with (34) implies that, for $x \in(0,1)$, we have

$$
G b(x) \approx x^{\min (2,4-\beta)}(1-x)^{\min (2,4-\gamma)} \psi_{\beta}(x) \phi_{\gamma}(1-x) .
$$

\section{Proof of Theorem 1}

In order to prove Theorem 1, we need the following lemma.

Lemma 13. Assume that the function a satisfies $\left(H_{0}\right)$ and put $\omega(t)=a(t)\left(\theta_{\lambda, \mu}(t)\right)^{\sigma}$ for $t \in(0,1)$. Then, one has, for $x \in(0,1)$,

$$
G \omega(x) \approx \theta_{\lambda, \mu}(x)
$$

Proof. Put $r=\min (2,(4-\lambda) /(1-\sigma))$ and $s=\min (2,(4-$ $\mu) /(1-\sigma))$. Then, for $t \in(0,1)$, we have

$$
\begin{aligned}
\omega(t)= & t^{-\lambda+r \sigma} L_{1}(t)\left(\widetilde{L}_{1}(t)\right)^{\sigma /(1-\sigma)}(1-t)^{-\mu+s \sigma} \\
& \times L_{2}(1-t)\left(\widetilde{L}_{2}(1-t)\right)^{\sigma /(1-\sigma)} .
\end{aligned}
$$

Let $\beta=\lambda-r \sigma, \gamma=\mu-s \sigma, L_{3}(t)=L_{1}(t)\left(\widetilde{L}_{1}(t)\right)^{\sigma /(1-\sigma)}$, and $L_{4}(t)=L_{2}(t)\left(\widetilde{L}_{2}(t)\right)^{\sigma /(1-\sigma)}$. Then, using Proposition 12, we obtain by a simple computation that

$$
G(\omega)(x) \approx \theta_{\lambda, \mu}(x)
$$

Proof of Theorem 1. From Lemma 13, there exists $M>1$ such that for each $x \in(0,1)$

$$
\frac{1}{M} \theta_{\lambda, \mu}(x) \leq G \omega(x) \leq M \theta_{\lambda, \mu}(x)
$$

where $\omega(t)=a(t)\left(\theta_{\lambda, \mu}(t)\right)^{\sigma}$. 


$$
\text { Put } \begin{aligned}
c_{0} & =M^{1 /(1-|\sigma|)} \text { and let } \\
\Lambda & =\left\{u \in C([0,1]): \frac{1}{c_{0}} \theta_{\lambda, \mu} \leq u \leq c_{0} \theta_{\lambda, \mu}\right\} .
\end{aligned}
$$

In order to use a fixed point theorem, we define the operator $T$ on $\Lambda$ by

$$
T u(x)=G\left(a u^{\sigma}\right)(x)=\int_{0}^{1} G(x, t) a(t) u^{\sigma}(t) d t .
$$

For this choice of $c_{0}$, we can easily prove that, for $u \in \Lambda$, we have $T u \leq c_{0} \theta_{\lambda, \mu}$ and $T u \geq\left(1 / c_{0}\right) \theta_{\lambda, \mu}$.

Now, since the function $(x, t) \rightarrow G(x, t)$ is continuous on $[0,1] \times[0,1]$ and, by Proposition 9, Corollary 10, and Lemma 13, the function $t \rightarrow t^{2}(1-t)^{2} a(t) \theta_{\lambda, \mu}^{\sigma}(t)$ is integrable on $(0,1)$, we deduce that the operator $T$ is compact from $\Lambda$ to itself. It follows by the Schauder fixed point theorem that there exists $u \in \Lambda$ such that $T u=u$. Then, $u \in C([0,1])$ and $u$ satisfies the equation

$$
u(x)=G\left(a u^{\sigma}\right)(x) .
$$

Since the function $t \rightarrow t^{2}(1-t)^{2} a(t) u^{\sigma}(t)$ is continuous and integrable on $(0,1)$, then by Proposition 11 , the function $u$ is a positive solution in $C^{4}((0,1)) \cap C([0,1])$ of problem $(1)$.

Finally, let us prove that $u$ is the unique positive continuous solution satisfying (9). To this aim, we assume that (1) has two positive solutions $u, v \in C^{4}((0,1)) \cap C([0,1])$ satisfying (9) and consider the nonempty set $J=\{m \geq 1: 1 / m \leq u / v \leq$ $m\}$ and put $c=\inf J$. Then, $c \geq 1$ and we have $(1 / c) v \leq u \leq c v$. It follows that $u^{\sigma} \leq c^{|\sigma|} v^{\sigma}$ and consequently

$$
\begin{aligned}
\left(c^{|\sigma|} v-u\right)^{(4)}= & a\left(c^{|\sigma|} v^{\sigma}-u^{\sigma}\right):=f \geq 0 \\
\left(c^{|\sigma|} v-u\right)(0) & =\left(c^{|\sigma|} v-u\right)(1) \\
& =\left(c^{|\sigma|} v-u\right)^{\prime}(0) \\
& =\left(c^{|\sigma|} v-u\right)^{\prime}(1)=0 .
\end{aligned}
$$

Since the function $t \rightarrow t^{2}(1-t)^{2} f(t)$ is continuous and integrable on $(0,1)$, it follows by Proposition 11 that $c^{|\sigma|} v-$ $u=G\left(a\left(c^{|\sigma|} v^{\sigma}-u^{\sigma}\right)\right) \geq 0$. By symmetry, we obtain also that $v \leq c^{|\sigma|} u$. Hence, $c^{|\sigma|} \in J$ and $c \leq c^{|\sigma|}$. Since $|\sigma|<1$, then $c=1$ and consequently $u=v$.

Example 14. Let $\sigma \in(-1,1)$ and let $a$ be a positive continuous function on $(0,1)$ such that

$$
a(t) \approx t^{-\lambda}(1-t)^{-\mu} \log \left(\frac{2}{1-t}\right),
$$

where $\lambda<3+\sigma$ and $\mu<3+\sigma$. Then, using Theorem 1, problem (1) has a unique positive continuous solution $u$ satisfying the following estimates:

$$
\begin{aligned}
u(x) \approx & x^{\min (2,(4-\lambda) /(1-\sigma))}\left(\widetilde{L}_{1}(x)\right)^{1 /(1-\sigma)} \\
& \times(1-x)^{\min (2,(4-\mu) /(1-\sigma))}\left(\widetilde{L}_{2}(1-x)\right)^{1 /(1-\sigma)},
\end{aligned}
$$

where

$$
\begin{gathered}
\widetilde{L}_{1}(x)= \begin{cases}1 & \text { if } \lambda \neq 2(1+\sigma), \\
\log \left(\frac{2}{x}\right) & \text { if } \lambda=2(1+\sigma),\end{cases} \\
\widetilde{L}_{2}(x)= \begin{cases}1 & \text { if } \mu<2(1+\sigma), \\
\left(\log \left(\frac{2}{x}\right)\right)^{2} & \text { if } \mu=2(1+\sigma), \\
\log \left(\frac{2}{x}\right) & \text { if } 2(1+\sigma)<\mu<3+\sigma .\end{cases}
\end{gathered}
$$

\section{Conflict of Interests}

The author declares that there is no conflict of interests regarding the publication of this paper.

\section{References}

[1] C. Bai, "Existence of positive solutions for fourth-order threepoint boundary value problems," Boundary Value Problems, vol. 2007, Article ID 68758, 10 pages, 2007.

[2] Y. Cui and Y. Zou, "Positive solutions of singular fourth-order boundary-value problems," Electronic Journal of Differential Equations, vol. 2006, no. 39, pp. 1-10, 2006.

[3] N. Kosmatov, "Countably many solutions of a fourth order boundary value problem," Electronic Journal of Qualitative Theory of Differential Equations, no. 12, pp. 1-15, 2004.

[4] R. Ma and C. C. Tisdell, "Positive solutions of singular sublinear fourth-order boundary value problems," Applicable Analysis, vol. 84, no. 12, pp. 1199-1220, 2005.

[5] D. O'Regan, "Solvability of some fourth (and higher) order singular boundary value problems," Journal of Mathematical Analysis and Applications, vol. 161, no. 1, pp. 78-116, 1991.

[6] M. Pei and S. K. Chang, "Monotone iterative technique and symmetric positive solutions for a fourth-order boundary value problem," Mathematical and Computer Modelling, vol. 51, no. 910, pp. 1260-1267, 2010.

[7] Q. Yao, "Positive solutions for eigenvalue problems of fourthorder elastic beam equations," Applied Mathematics Letters, vol. 17, no. 2, pp. 237-243, 2004.

[8] Y. Liu and G. Weigao, "Double positive solutions of fourthorder nonlinear boundary value problems," Applicable Analysis, vol. 82, no. 4, pp. 369-380, 2003.

[9] K. Zhang and C. Wang, "The existence of positive solutions of a class of fourth-order singular boundary value problems," Acta Mathematica Scientia. Series A, vol. 29, no. 1, pp. 127-135, 2009.

[10] V. Maric, Regular Variation and Differential Equations, vol. 1726 of Lecture Notes in Mathematics, Springer, Berlin, Germany, 2000.

[11] E. Seneta, Regularly Varying Functions, vol. 508 of Lecture Notes in Mathematics, Springer, Berlin, Germany, 1976.

[12] R. Chemmam, H. Mâagli, S. Masmoudi, and M. Zribi, "Combined effects in nonlinear singular elliptic problems in a bounded domain," Advances in Nonlinear Analysis, vol. 1, no. 4, pp. 301-318, 2012.

[13] N. Zeddini, R. Alsaedi, and H. Maagli, "Exact boundary behavior of the unique positive solution to some singular elliptic problems," Nonlinear Analysis: Theory, Methods \& Applications, vol. 89, pp. 146-156, 2013. 


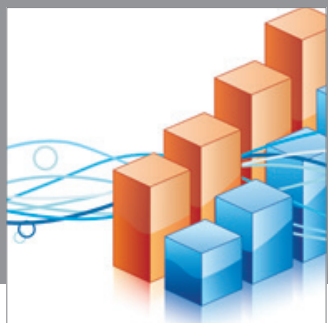

Advances in

Operations Research

mansans

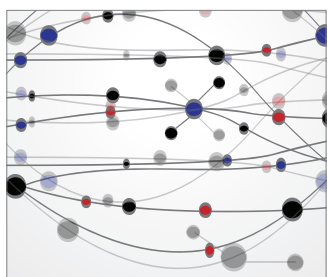

The Scientific World Journal
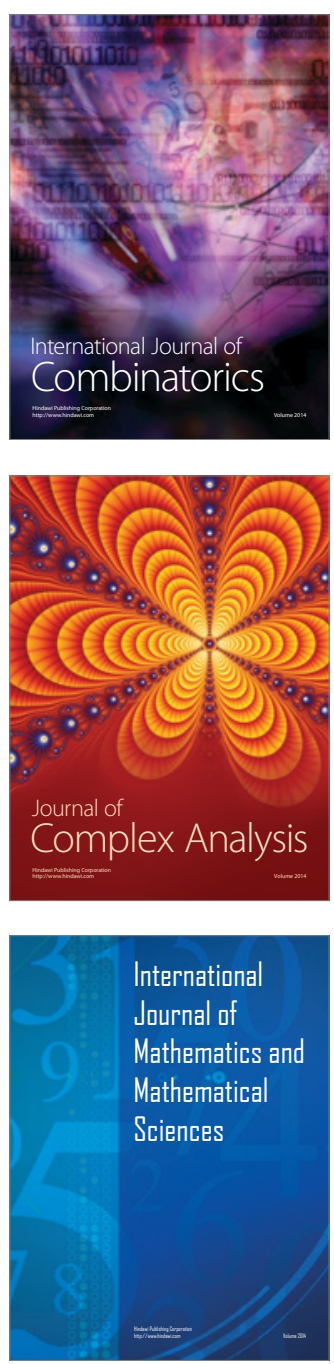
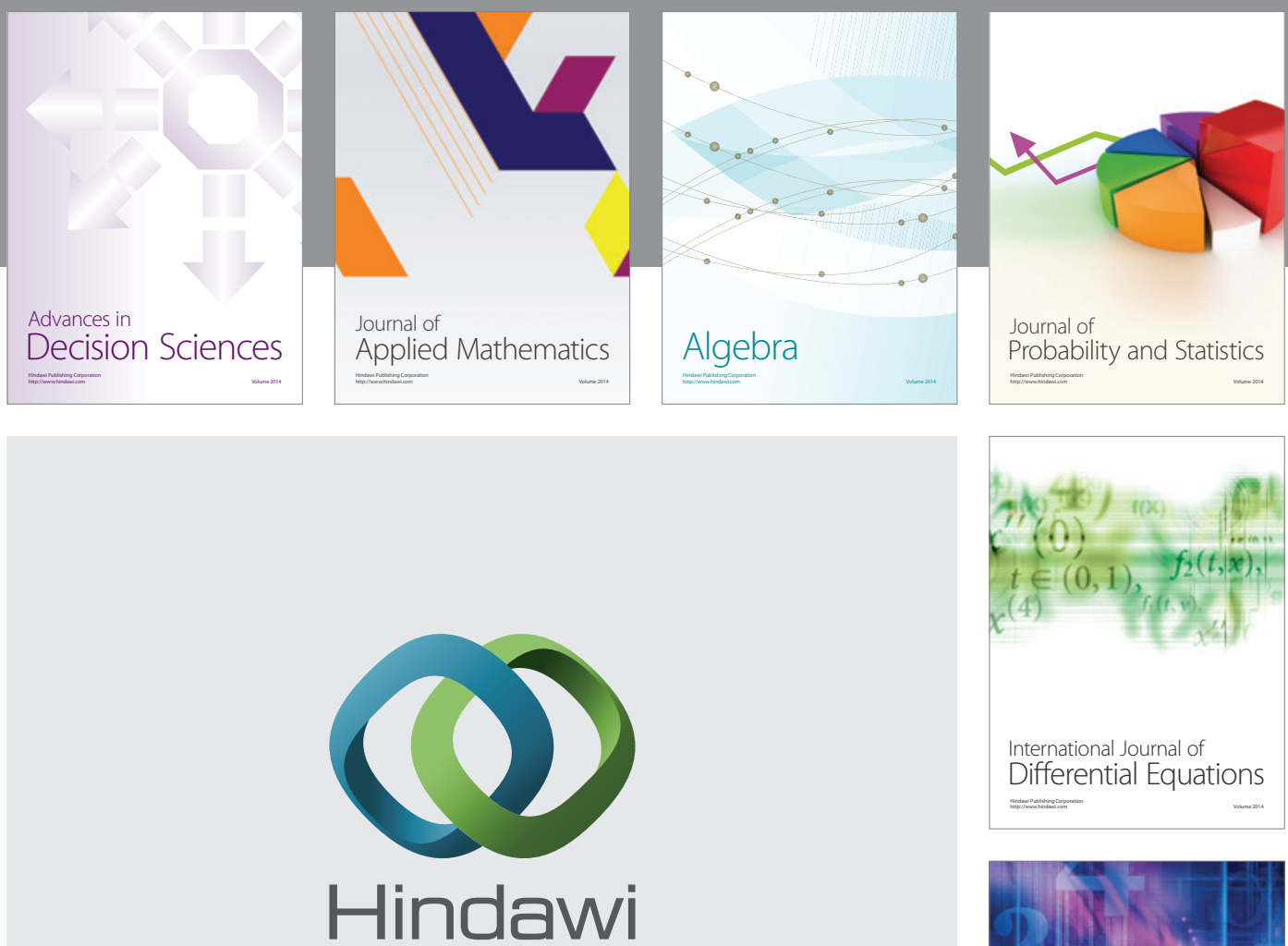

Submit your manuscripts at http://www.hindawi.com
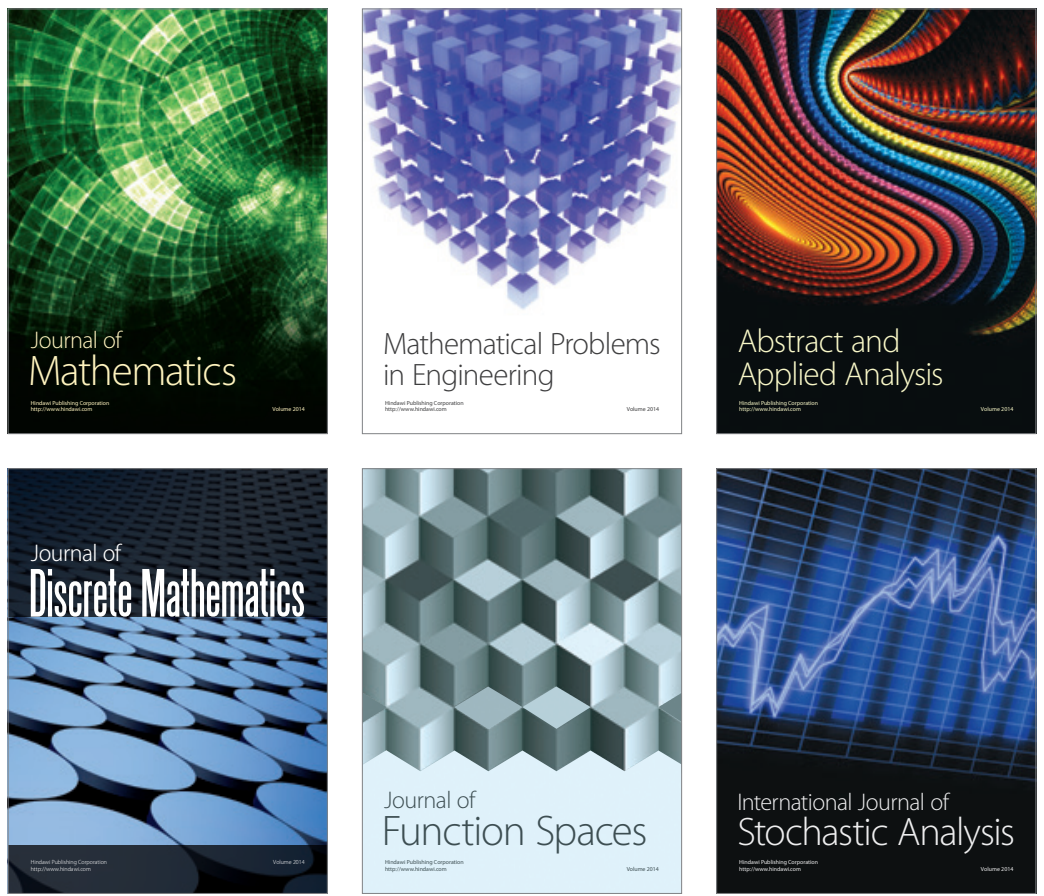

Journal of

Function Spaces

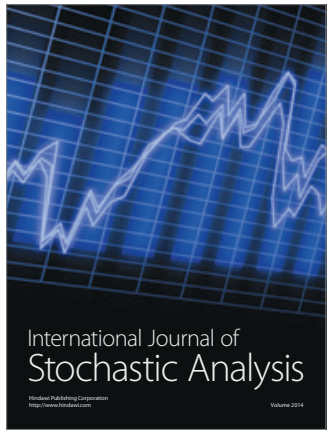

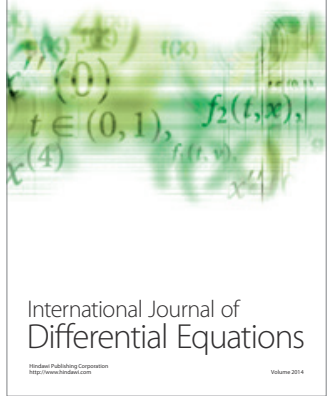
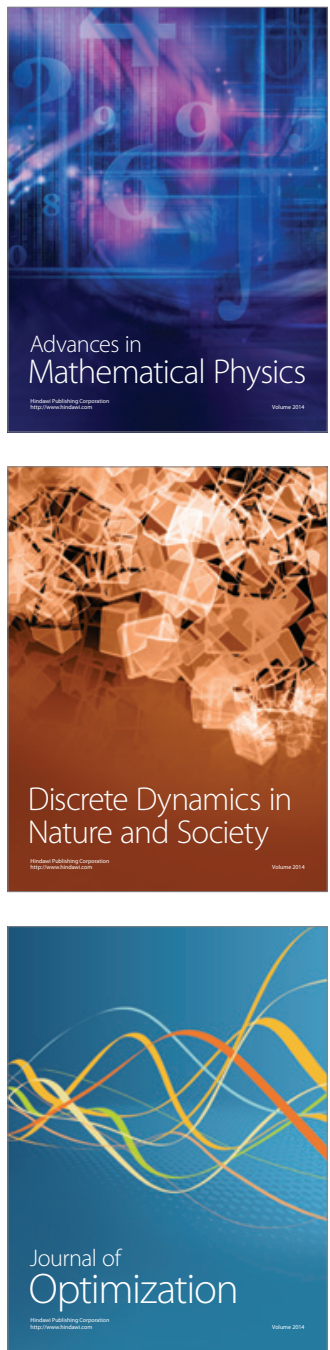